\title{
DIFERENCIAS CLÍNICAS Y POLISOMNOGRÁFICAS ENTRE OBESOS Y NO OBESOS CON SÍNDROME DE APNEAS-HIPOPNEAS DEL SUEÑO
}

\author{
Jorge Rey de Castro ${ }^{1,2,3, a}$, Edmundo Rosales-Mayor ${ }^{2,4, b}$
}

\begin{abstract}
RESUMEN
Objetivos. Describir las diferencias clínicas y polisomnográficas en pacientes obesos y no obesos con diagnóstico del síndrome de apneas-hipopneas del sueño (SAHS). Materiales y métodos. A los pacientes incluidos se les realizó un examen físico, se aplicó la escala de somnolencia de Epworth (ESE) y además se les realizó una polisomnografía. Se consideró obeso si el índice de masa corporal (IMC) era mayor o igual a $30 \mathrm{~kg} / \mathrm{m}^{2}$. Resultados. Se analizaron 408 pacientes con SAHS, de estos, 119 (47 \%) fueron obesos. El SAHS fue leve en 101 (25\%), moderado en 91 (22\%) y severo en 216 (53 \%). No hubo diferencias por sexo, edad y puntaje ESE al compararse obesos con no obesos. La diferencias del perímetro cervical y presencia de somnolencia según ESE fue significativamente mayor en obesos. Estos tuvieron mayor comorbilidad en términos de dislipidemia, hipertensión arterial y enfermedad coronaria. De las variables polisomnográficas, los índices de eventos respiratorios fueron mayores en obesos, asimismo fueron peores los valores de saturación de oxígeno. No hubo diferencias en el resto de variables. El análisis de regresión mostró asociación entre la obesidad y la severidad del SAHS. Conclusiones. EI SAHS no es una enfermedad limitada a la población obesa, aunque esta última tienen mayor comorbilidad y formas más severas de enfermedad.
\end{abstract}

Palabras clave: Apnea del sueño obstructiva; Obesidad; Transtornos por excesiva somnolencia; Polisomnografía; Perú (fuente: DeCS BIREME).

\section{CLINICAL AND POLYSOMNOGRAPHYC DIFFERENCES BETWEEN OBESE AND NON-OBESE PATIENTS WITH OBSTRUCTIVE SLEEP APNEA-HYPOPNEA SYNDROME}

\begin{abstract}
Objectives. To describe the clinical and polysomnographyc differences between obese and non- obese patients with obstructive sleep apnea-hypopnea (OSAH) syndrome. Materials and methods. A physical examination, the Epworth Sleepiness Scale (ESS) and a polysomnography were performed to all included patients. Obesity was defined as a body mass index (BMI) of $\geq 30 \mathrm{~kg} / \mathrm{m} 2$. Results. 408 patients with OSAH were analyzed, out of these, $119(47 \%)$ were obese. OSAH was mild in 101 (25\%), moderate in 91 (22\%) and severe in 216 (53\%). There were no age, sex and EES score differences between obese and non-obese patients. Cervical perimeter and sleepiness prevalence according to the ESS were significant higher in obese population. Obese patients had higher comorbidity related to dyslipidemia, high blood pressure and coronary disease. With respect to the polysomnographyc variables, the indexes of respiratory events and the levels of oxygen saturation were worse in obese patients. There were no differences in the other variables. The regression analysis showed association between obesity and OSAH severity. Conclusions. OSAH is a clinical condition not limited to obese population, although this group has a higher comorbidity and OSAH severity.
\end{abstract}

Key words: Sleep apnea, obstructive; Obesity; Disorders of excessive somnolence; Polisomnography, Peru (source: MeSH NLM).

\footnotetext{
CENTRES-Clínica Anglo Americana. Lima, Perú.

Grupo de Investigación en Sueño. Lima, Perú.

Universidad Peruana Cayetano Heredia. Lima, Perú.

Instituto Clínic del Tórax, Hospital Clínic de Barcelona. Barcelona, España.

a Médico Neumólogo y Trastornos Respiratorios del Sueño, Magister en Medicina; b Médico Cirujano, Magister en Medicina, Máster en sueño: fisiología y medicina
}

Recibido: 08-08-11 Aprobado: 12-10-11 


\section{INTRODUCCIÓN}

Los trastornos respiratorios del sueño constituyen un grupo frecuente de enfermedades en la práctica médica. La clasificación, propuesta por la American Academy of Sleep Medicine (AASM), de estas enfermedades, incluye al síndrome de apneas-hipopneas del sueño (SAHS), síndrome de apnea central, síndrome de hipoventilación con hipoxemia y una miscelánea ${ }^{(1)}$. En cuanto al SAHS, estudios epidemiológicos de prevalencia importantes han determinado cifras que fluctúan entre 2 a $6 \%$ en población general, mientras que en población de 70 a 100 años una prevalencia entre 15 a $26 \%{ }^{(2-4)}$. La enfermedad es causada por eventos obstructivos parciales o totales de la vía aérea alta durante el dormir, que llevan a una desaturación de oxígeno intermitente, despertares electroencefalográficos, fragmentación del sueño, somnolencia diurna, cambios neurocognitivos y mala calidad de vida ${ }^{(5)}$. Los síntomas más frecuentes son ronquido intenso, pausas respiratorias durante el sueño con respiración asfixiante y somnolencia diurna. Existe amplia documentación sobre que el SAHS es un factor de riesgo de hipertensión arterial, enfermedad cardio y cerebro vascular, accidentes de tránsito durante la conducción debido a somnolencia y muerte causada tanto por infarto de miocardio como accidente cerebro vascular ${ }^{(6)}$.

Está reconocido que la obesidad es el factor de riesgo más importante para desarrollar SAHS en población adulta de la edad media de la vida, el incremento moderado del peso corporal aumenta el riesgo y está documentada la relación aritmética entre el incremento o disminución del peso y la variación respectiva del índice de apnea hipopnea (IAH) ${ }^{(7)}$. De otro lado, el incremento de la obesidad acelera la progresión de la enfermedad ${ }^{(8)}$. Los cambios de estilo de vida y la dieta con bajo contenido calórico constituyen el tratamiento de primera línea en pacientes con SAHS leve ${ }^{(7)}$.

El objetivo de esta investigación es describir las diferencias del comportamiento clínico y polisomnográfico en pacientes obesos y no obesos con diagnóstico documentado de SAHS.

\section{MATERIALES Y MÉTODOS}

La población reclutada para el estudio forma parte de la cohorte del Centro de Trastornos Respiratorios del Sueño (CENTRES) de la ClínicaAnglo Americana en Lima-Perú, todas las variables fueron registradas prospectivamente en una base de datos del programa Access ${ }^{\mathrm{TM}}$ versión 11 (Microsoft Corp, Redmond, Washington, EEUU). Antes de ingresar a la entrevista médica, los pacientes firmaron el consentimiento informado y luego respondieron la escala de somnolencia de Epworth - versión peruana modificada (ESE-VPM) ${ }^{9}$. La entrevista médica y el examen físico fue realizado por un especialista en medicina del sueño (JRC).

Entre los 15 a 30 días posteriores a la consulta se practicó una polisomnografía (PSG) supervisada, para ello se usó el equipo Easy II@ de la Cadwell Inc. y EB Neuro B.E. Light. El arreglo polisomnográfico empleado fue: EEG (C3-A2, C4-A1, 01-A2, 02-A1), electro oculograma (EOG) bilateral, electromiografía del mentón, flujo oronasal con thermistor, cánula nasal de presión (dispositivo PTAF de Pro-Tech ${ }^{\mathrm{TM}}$ ), micrófono de ronquido en el área pretraqueal, bandas de esfuerzo torácico y abdominal, oximetría de pulso, electrocardiograma en derivación II, electromiografía de músculos tibiales anteriores en ambas extremidades y posición corporal. De acuerdo con la clasificación de la denominada American Sleep Disorders Association (ASDA) esta prueba de monitoreo corresponde a estudio de nivel I ( ${ }^{(10)}$. Para los efectos de la clasificación de los estadios del sueño se empleó la descripción original de Rechtschaffen y Kales ${ }^{(11)}$.

Se excluyeron pacientes con diagnóstico previo de SAHS que usaban modalidades terapéuticas tales como prótesis de avance mandibular o CPAP, pacientes operados de uvulopalatofaringoplastía, otra enfermedad asociada al sueño por historia clínica o polisomnografía, con trastornos del ciclo circadiano, con durmientes diurnos y cualquier condición física (enfermedades neuromusculares, deformación de caja torácica, alteraciones anatómicas de la vía aérea derivada de accidentes o intervenciones quirúrgicas) o psicológica (depresión mayor, psicosis, trastorno bipolar o trastorno obsesivo compulsivo) que causara sesgos en los resultados.

\section{DEFINICIONES}

La apnea fue definida como cese completo o casi completo (mayor o igual al $90 \%$ ) de la señal del termistor y la cánula nasal con una duración igual o mayor de diez segundos acompañada de una desaturación mayor al $3 \%$ o despertar (arousal) respiratorio de acuerdo con los criterios de la ASDA ${ }^{(12)}$. La hipopnea fue definida como una reducción discernible (mayor o igual al $50 \%$ ) de la señal del termistor, o de la cánula nasal igual o mayor de diez segundos acompañada de una desaturación mayor del $3 \%$ o arousal respiratorio. Los episodios respiratorios fueron obstructivos si se acompañaban de esfuerzo tóraco-abdominal, centrales si estaba ausente y mixtos cuando coexistían ambos, comenzando por el componente de tipo central y finalizando con el 
obstructivo. Los esfuerzos respiratorios asociados a microdespertares (ERAM) también llamados RERAS se definieron de acuerdo con los criterios de la AASM ${ }^{(13)}$.

EI IAH fue calculado sumando las apneas e hipopneas y dividiendo entre el número total de horas de sueño. El índice de disturbio respiratorio (IDR) fue calculado por la sumatoria de apneas, hipopneas y ERAM dividiendo entre el número total de horas de sueño. El nadir de la saturación nocturna de oxígeno o desaturación máxima (DesatMax) como el valor mínimo de oximetría de pulso alcanzado durante el sueño. Tiempo porcentual de sueño con saturación de oxígeno por debajo de 90 \% (T90), definido como el cociente entre el tiempo en minutos con valores de saturación bajo $90 \%$ dividido entre el tiempo total en minutos de sueño y luego multiplicado por 100. Toda la calificación de los estadios de sueño y eventos respiratorios se realizaron en forma manual.

Se estableció la categoría hipertensión arterial (HTA) de acuerdo con los criterios del séptimo reporte de la Joint National Committee si la presión arterial sistólica era $\geq 140 \mathrm{mmHg}$ o la presión arterial diastólica $\geq 90 \mathrm{mmHg}$ o si el paciente acudia a la consulta empleando al menos un medicamento para controlar la hipertensión ${ }^{(14)}$. La dislipidemia fue definida de acuerdo con las pautas de Fredrickson et al. ${ }^{(15)}$ y la insuficiencia cardiaca de acuerdo con la clasificación funcional de la Asociación Cardiológica de Nueva York ${ }^{(16)}$. Enfermedad coronaria como historia de angina o infarto al miocardio o colocación de por lo menos un stent coronario o cirugía de puentes aorto-coronarios.

Los estudios de PSG fueron de noche partida (PSGNP) y noche completa (PSG-NC). En PSG-NP se indicó titulación del CPAP (Continuous Positive Airway Pressure o presión positiva continua en la vía aérea) en la segunda parte del estudio si el paciente cumplía con uno de los siguientes criterios: IAH 20 o más por hora o 40 eventos obstructivos en dos horas o eventos obstructivos con desaturación asociada con arritmias malignas (fenómeno $\mathrm{R}$ sobre $\mathrm{T}$, taquicardia ventricular polimórfica o sostenida y fibrilación ventricular). $\mathrm{Si}$ el paciente no cumplía los criterios señalados se continuaba el registro nocturno sin interrupciones, por lo que este tipo de polisomnografía se calificó como PSGNC. Basándonos en las publicaciones de Sanders ${ }^{(17)}$ y Khawaja ${ }^{(18)}$, los pacientes sometidos a una PSG-NP o PSG-NC fueron analizados de forma conjunta.

En el presente estudio el diagnóstico de SAHS fue establecido de acuerdo con los criterios de la AASM si IAH fue $\geq 5$ por hora de sueño (1). EI SAHS fue leve si el $\mathrm{IAH}$ fue de 5 a 14 , moderado si de 15 a 29 y severo si $\mathrm{IAH} \geq 30$.
Se utilizó una balanza y un tallímetro calibrados para calcular el índice de masa corporal (IMC) en unidades de $\mathrm{kg} / \mathrm{m}^{2}$. Un IMC $\geq 30 \mathrm{~kg} / \mathrm{m}^{2}$ se consideró como obesidad y un IMC $<30 \mathrm{~kg} / \mathrm{m}^{2}$ como no obesidad. Empleamos la técnica propuesta por Lohman ${ }^{(19)}$ para la medición del perímetro cervical $(P C)$.

\section{ESCALA DE SOMNOLENCIA DE EPWORTH}

La escala de somnolencia Epworth es una herramienta que evalúa la somnolencia de manera subjetiva, fue diseñada por Murray W. Johns ${ }^{(20)}$ en 1991. Consiste en un breve cuestionario que pregunta por la probabilidad de quedarse dormido en ocho distintas situaciones de la vida cotidiana. Se empleó una validación cultural peruana de la escala y una versión peruana modificada del mismo instrumento para población que no conduce vehículos, denominada ESE-VPM ${ }^{(9)}$.

\section{ANÁLISIS ESTADÍSTICO}

Se empleó el programa informático Stata 10.0 (STATA Corp, College Station, Texas, EEUU) para el análisis estadístico. La información fue analizada como $\mathrm{N}$ (porcentual) en las variables categóricas y con la media \pm desviación estándar en las variables continuas. Se calculó el chi cuadrado para comparar variables categóricas. Para la comparación de dos medias se empleó la prueba $\mathrm{t}$ de Student. En la comparación de las medias de más de dos muestras se realizó análisis de varianza (ANOVA). La correlación de Pearson fue empleada para calcular el grado de asociación.

Se realizó un análisis de regresión lineal múltiple considerando el IAH como variable dependiente y como variable independiente la presencia o no de obesidad. Se estableció previamente que otras variables independientes fueran la edad, sexo, PC, ESD y la presencia de comorbilidades (HTA, enfermedad coronaria, insuficiencia cardiaca o dislipidemia). Se consideró significativo un valor de $p<0,05$. El Comité de Bioética Institucional de la Clínica Anglo Americana autorizó la implementación del presente estudio.

\section{RESULTADOS}

Durante el período de evaluación entre julio de 1995 y noviembre de 2010 se registró en la base de datos, una población inicial de 651 pacientes de los cuales 100 (15\%) fueron mujeres. Fueron excluidos 137 casos. Con ello quedaron 514 casos, 106 sin SAHS y 408 con SAHS. Esta última muestra constituyó el universo de la población que ingresó al análisis (Figura 1). 


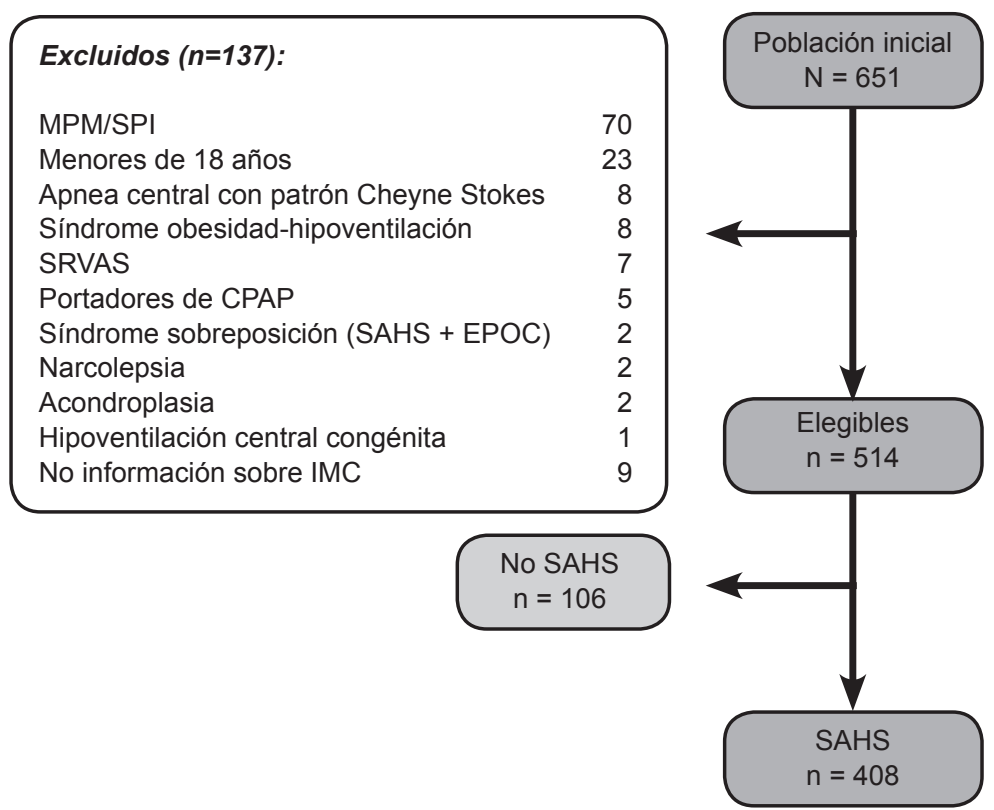

Figura 1. Flujograma de participantes en el estudio.

SRVAS: síndrome de resistencia vía aérea superior. MPM: mioclonías periódicas de los miembros. SPI: síndrome de piernas de inquietas. SAHS: síndrome de apnea-hipopneas del sueño. EPOC: enfermedad pulmonar obstructiva crónica. CPAP: presión positiva continua en vía aérea. IMC: índice de masa corporal.

Treinta y ocho (9\%) fueron de sexo femenino, la edad promedio fue de 49,4 $\pm 12,1$ años, y el IMC promedio fue de $30,3 \pm 4,6 \mathrm{~kg} / \mathrm{m}^{2}$.

El SAHS fue leve en 101 (25\%), moderado en $91(22 \%)$ y severo en 216 (53\%). Ciento diecinueve pacientes tenían obesidad (47\%). La comparación de variables clínica y polisomnográficas entre pacientes con obesidad frente a sin obesidad se muestran en la Tabla 1. Las formas severas de SAHS fueron más frecuentes en pacientes con obesidad (68\% frente a $40 \%$; $p<0,001$ ).

El análisis de correlación de Pearson del IMC con las siguientes variables: PC, ESE-VPM, Índice de despertares, IAH, DesatMax y T90; tanto en la población de no obesos y en obesos se muestran en la Tabla 2. Los resultados del análisis de regresión lineal múltiple se muestran en la Tabla 3.

\section{DISCUSIÓN}

La obesidad es el principal factor de riesgo de SAHS en la población adulta ${ }^{(2)}$. De acuerdo con la literatura médica, aproximadamente dos terceras partes de los pacientes con SAHS tiene obesidad (21) mientras que la prevalencia de SAHS en obesos mórbidos es de $50 \%{ }^{(22)}$. La hipótesis planteada estriba en que particularmente los pacientes con obesidad central tienen acumulación de tejido graso en la lengua, cara posterior-lateral de
Tabla 1. Comparación de variables clínicas y polisomnográficas en pacientes con y sin obesidad.

\begin{tabular}{|c|c|c|c|}
\hline & Obesos & No obesos & $p$ \\
\hline Sexo femenino & $7,9 \%$ & $10,6 \%$ & 0,357 \\
\hline Edad (años) & $48,9 \pm 11,2$ & $49,8 \pm 12,8$ & 0,462 \\
\hline Perímetro cervical & $44,6 \pm 3,9$ & $40,3 \pm 3,3$ & $<0,001$ \\
\hline $\begin{array}{l}\text { Escala somnolencia } \\
\text { Epworth (ESE) }\end{array}$ & $11,1 \pm 5,5$ & $10,0 \pm 5,0$ & 0,053 \\
\hline $\begin{array}{l}\text { Excesiva somnolencia } \\
\text { diurna }(E S E>10)\end{array}$ & $52,1 \%$ & $41,3 \%$ & 0,029 \\
\hline Dislipidemia & $39,5 \%$ & $20,2 \%$ & $<0,001$ \\
\hline Hipertensión arterial & $71,6 \%$ & $37,2 \%$ & $<0,001$ \\
\hline Enfermedad coronaria & $8,9 \%$ & $3,7 \%$ & 0,027 \\
\hline Insuficiencia cardiaca & $1,1 \%$ & $0,0 \%$ & 0,129 \\
\hline $\begin{array}{l}\text { Latencia de sueño } \\
\text { (min) }\end{array}$ & $9,9 \pm 14,9$ & $10,2 \pm 17,7$ & 0,843 \\
\hline $\begin{array}{l}\text { Latencia de sueño } \\
\text { REM (min) }\end{array}$ & $106,2 \pm 54,6$ & $107,1 \pm 61,6$ & 0,896 \\
\hline Índice de despertares & $51,9 \pm 28,8$ & $42,2 \pm 25,1$ & $<0,001$ \\
\hline $\begin{array}{l}\text { Índice de apnea } \\
\text { hipopnea (IAH) }\end{array}$ & $48,7 \pm 29,1$ & $28,2 \pm 20,2$ & $<0,001$ \\
\hline IAH en REM & $42,9 \pm 24,4$ & $27,2 \pm 23,1$ & $<0,001$ \\
\hline IAH en No-REM & $47,7 \pm 31,7$ & $27,4 \pm 21,2$ & $<0,001$ \\
\hline IAH en posición lateral & $39,5 \pm 32,8$ & $19,3 \pm 20,2$ & $<0,001$ \\
\hline IAH en posición supina & $59,5 \pm 28,6$ & $42,0 \pm 25,2$ & $<0,001$ \\
\hline Índice de ERAM & $4,9 \pm 6,6$ & $5,6 \pm 6,5$ & 0,449 \\
\hline $\begin{array}{l}\text { Índice de disturbio } \\
\text { respiratorio }\end{array}$ & $38,9 \pm 23,5$ & $29,2 \pm 17,9$ & $<0,001$ \\
\hline Desaturación máxima & $79,6 \pm 8,9$ & $84,6 \pm 6,4$ & $<0,001$ \\
\hline T90 & $15,9 \pm 20,0$ & $5,0 \pm 10,4$ & $<0,001$ \\
\hline
\end{tabular}

Los valores se presentan como porcentaje o media \pm desviación estándar. ERAM: eventos respiratorios asociados a microdespertares.

T90: tiempo porcentual de sueño con saturación de oxígeno debajo de $90 \%$. REM: rapid eye moviment. 
Tabla 2. Análisis de correlación de Pearson con algunas variables en paciente con y sin obesidad.

\begin{tabular}{|c|c|c|c|}
\hline & No obesos & Obesos & Todos \\
\hline Perímetro cervical & $\begin{array}{c}0,571 \\
(p<0,001)\end{array}$ & $\begin{array}{c}0,553 \\
(p<0,001)\end{array}$ & $\begin{array}{c}0,694 \\
(p<0,001)\end{array}$ \\
\hline $\begin{array}{l}\text { Escala de somnolencia } \\
\text { de Epworth }\end{array}$ & $\begin{array}{c}-0,035 \\
(p=0,621)\end{array}$ & $\begin{array}{c}0,179 \\
(p=0,016)\end{array}$ & $\begin{array}{c}0,139 \\
(p=0,006)\end{array}$ \\
\hline Índice de despertares & $\begin{array}{c}0,168 \\
(p=0,014)\end{array}$ & $\begin{array}{c}0,162 \\
(p=0,029)\end{array}$ & $\begin{array}{c}0,236 \\
(p<0,001)\end{array}$ \\
\hline $\begin{array}{l}\text { Índice de Apnea } \\
\text { Hipopnea }\end{array}$ & $\begin{array}{c}0,269 \\
(p<0,001)\end{array}$ & $\begin{array}{c}0,377 \\
(p<0,001)\end{array}$ & $\begin{array}{c}0,497 \\
(p<0,001)\end{array}$ \\
\hline Desaturación máxima & $\begin{array}{c}-0,241 \\
(p<0,001)\end{array}$ & $\begin{array}{c}-0,135 \\
(p=0,067)\end{array}$ & $\begin{array}{c}-0,341 \\
(p<0,001)\end{array}$ \\
\hline $\begin{array}{l}\text { Tiempo porcentual de } \\
\text { sueño con saturación } \\
\text { de oxígeno por debajo } \\
\text { de } 90 \%\end{array}$ & $\begin{array}{c}0,189 \\
(p=0,005)\end{array}$ & $\begin{array}{c}0,312 \\
(p<0,001)\end{array}$ & $\begin{array}{c}0,425 \\
(p<0,001)\end{array}$ \\
\hline
\end{tabular}

Valores que se muestran: $\mathrm{R}$ de Pearson (valor $\mathrm{p}$ ).

la faringe y paladar blando. Ello conduce a la pérdida del tono muscular, disminución del área de sección de la faringe aumentando la presión extraluminal y, finalmente, colapso de la vía aérea superior (VAS) ${ }^{(23)}$. De otro lado, la disminución del volumen pulmonar altera las propiedades elásticas del pulmón lo cual agrava la propensión al colapso en la VAS ${ }^{(24)}$. Sin embargo, los pacientes sin obesidad no tienen estas características antropomórficas y como ya ha sido señalado el SAHS no es exclusivo de obesos ${ }^{(25-26)}$.

Sakakibara et al. (27) describieron las anormalidades cefalométricas en pacientes japoneses no obesos y obesos con SAHS, encontrando que estas eran similares a las descritas en pacientes caucásicos. En los obesos el incremento de los tejidos blandos de la VAS cumplen un papel importante en la patogénesis del síndrome mientras que en no obesos la estructura ósea sería el factor dominante del SAHS. Li et al. ${ }^{(28)}$ describieron la estructura ósea cráneo-facial en asiáticos, la información obtenida sugiere que esta característica anatómica sería un factor de riesgo para desarrollar SAHS en esta población. En todos los pacientes incluidos en el estudio predominó el sexo masculino y las formas severas de SAHS. Estos resultados corresponden a una población sesgada en la medida que el análisis fue realizado a partir de enfermos evaluados selectivamente en un laboratorio de sueño.

No hubo diferencias por sexo, edad y puntaje promedio de la ESE-VPM al compararse obesos con no obesos. La diferencias del PC y presencia de ESD fue significativamente mayor en obesos. Estos tuvieron mayor comorbilidad en términos de dislipidemia, hipertensión arterial y enfermedad coronaria ${ }^{(7)}$.
Tabla 3. Análisis de regresión lineal múltiple para Índice apnea-hipopnea

\begin{tabular}{|c|c|c|c|c|}
\hline $\begin{array}{c}\text { Variables } \\
\text { independientes }\end{array}$ & Coeficiente & (IC $95 \%)$ & EE & $p$ \\
\hline Edad & $-0,2$ & $(-0,4-0,1)$ & 0,105 & 0,105 \\
\hline Sexo (varón) & 3,7 & $(-7,0-14,4)$ & 5,444 & 0,500 \\
\hline $\begin{array}{l}\text { Obesidad } \\
\left(\text { IMC } \geq 30 \mathrm{~kg} / \mathrm{m}^{2}\right)\end{array}$ & 10,3 & $(4,4-16,3)$ & 3,007 & 0,001 \\
\hline $\begin{array}{l}\text { Excesiva } \\
\text { somnolencia diurna } \\
\text { (ESE>10) }\end{array}$ & 5,3 & $(0,4-10,1)$ & 2,449 & 0,031 \\
\hline Perímetro cervical & 1,9 & $(1,0-2,8)$ & 0,445 & $<0,001$ \\
\hline Comorbilidades* & 4,0 & $(-1,5-9,5)$ & 2,803 & 0,153 \\
\hline
\end{tabular}

Las latencias del sueño y latencias REM fueron similares en los dos grupos y el valor del índice arousal fue mayor en obesos. Con relación a los eventos respiratorios propiamente dichos, el IAH en REM y NoREM, IAH posición supina y lateral e IAH de todo el estudio fue mayor en los obesos. No hubo diferencias en el índice ERAM. Análogamente el comportamiento de la saturación del oxígeno de la hemoglobina tuvo valores de DestMax y T90 más severos en obesos. En el análisis de regresión se encontró asociación de forma independiente entre la presencia de obesidad con la severidad del SAHS según el IAH.

La publicación de Chierakul et al. ${ }^{(29)}$, realizada en Bangkok, compara las características de los pacientes con SAHS sin y con obesidad. Las conclusiones destacan la función que cumple la estructura ósea en no obesos y, de otro lado, la mayor severidad en prácticamente todas las variables polisomnográficas cuantificadas en el grupo obesos. Si bien es cierto que en nuestra población no se emplearon medidas antropométricas, fue destacable la severidad de los resultados obtenidos por medio del monitoreo del sueño en obesos como el índice de arousal, IAH, DesatMax y T90. Estos resultados han sido descritos previamente por Sakakira ${ }^{(27)}$, Davies ${ }^{(30)}$ y sus respectivos colaboradores.

La ausencia en la cuantificación de variables antropométricas de los grupos estudiados y el sesgo de selección en pacientes evaluados en laboratorio del sueño fueron las principales limitaciones del presente estudio. El componente subjetivo de la herramienta utilizada (ESE-VPM), inherente a este tipo de cuestionarios constituyó también otra limitación del estudio. Finalmente, no es posible hacer inferencias con 
otras poblaciones ya que la muestra pertenece a una población clínica no probabilística.

En conclusión, se confirma que el SAHS no es una enfermedad limitada a la población obesa y que los obesos tienen mayor comorbilidad con formas más severas de enfermedad a juzgar por las variables polisomnográficas mencionadas.

\section{Contribuciones de autoría}

JRC y ERM participaron en la concepción del estudio, recolección de resultados, análisis e interpretación de datos, redacción del manuscrito, revisión crítica del artículo y la aprobación de la versión final a publicar. JRC contribuyó en el aporte de pacientes y material de estudio.

\section{Fuentes de financiamiento}

Autofinanciado.

\section{Conflictos de interés}

JRC es Director Científico de AGA S.A. en Perú. ERM declara no tener conflicto de interés.

\section{REFERENCIAS BIBLIOGRÁFICAS}

1. American Academy of Sleep Medicine. The international classification of sleep disorders: diagnostic and coding manual. 2nd ed. Westchester, IL: American Academy of Sleep Medicine; 2005.

2. Young T, Palta M, Dempsey J, Skatrud J, Weber S, Badr S. The occurrence of sleep-disordered breathing among middle-aged adults. N Engl J Med. 1993;328(17):1230-5.

3. Duran J, Esnaola S, Rubio R, Iztueta A. Obstructive sleep apnea-hypopnea and related clinical features in a population-based sample of subjects aged 30 to $70 \mathrm{yr}$. Am J Respir Crit Care Med. 2001;163(3 Pt 1):685-9.

4. Duran J, Esnaola S, Rubio R, De la Torre G. Obstructive sleep apnoea-hypopnoea in the elderly. A population-based study in the general population aged 71-100. European Respiratory J. 2000;16(Sup/31):167S.

5. Grupo Español de Sueño. Consenso nacional sobre Síndrome de Apneas-Hipopneas del Sueño. Arch Bronconeumol. 2005;41(Supl 4):1-110.

6. Rey de Castro J. Síndrome de Apneas Hipopneas del Sueño. ¿Asesino silencioso? Revisión de la literatura. Rev Med Hered. 2007;18(4):222-7.

7. Tuomilehto HP, Seppa JM, Partinen MM, Peltonen M, Gylling H, Tuomilehto JO, et al. Lifestyle intervention with weight reduction: first-line treatment in mild obstructive sleep apnea. Am J Respir Crit Care Med. 2009;179(4):320-7.

8. Young T, Skatrud J, Peppard PE. Risk factors for obstructive sleep apnea in adults. JAMA. 2004;291(16):2013-6.

9. Rosales-Mayor E, Rey de Castro J, Huayanay L, Zagaceta K. Validation and modification of the Epworth Sleepiness Scale in Peruvian population. Sleep Breath. 2011 Jan 30. [Epub ahead of print]
10. Ferber R, Millman R, Coppola M, Fleetham J, Murray $\mathbf{C F}$, Iber $\mathbf{C}$, et al. Portable recording in the assessment of obstructive sleep apnea. ASDA standards of practice. Sleep. 1994;17(4):378-92.

11. Rechtschaffen A, Kales AA. A manual of standardized terminology techniques and scoring system for sleep stages of human subjects. NIH Publication $\mathrm{N}^{\circ} 204$. Washington, DC: Government Printing Office; 1968.

12. American Sleep Disorders Association. EEG arousals: scoring rules and examples: a preliminary report from the Sleep Disorders Atlas Task Force of the American Sleep Disorders Association. Sleep. 1992;15(2):173-84.

13. Iber C, American Academy of Sleep Medicine. The AASM manual for the scoring of sleep and associated events: rules, terminology and technical specifications. Westchester, IL: American Academy of Sleep Medicine; 2007.

14. Chobanian AV, Bakris GL, Black HR, Cushman WC, Green LA, Izzo JL, Jr, et al. Seventh report of the Joint National Committee on Prevention, Detection, Evaluation, and Treatment of High Blood Pressure. Hypertension. 2003;42(6):1206-52.

15. Fredrickson DS, Levy RI, Lees RS. Fat transport in lipoproteins--an integrated approach to mechanisms and disorders. N Engl J Med. 1967;276(1):34-42.

16. Dolgin $\mathbf{M}$ (editor). Nomenclature and criteria for diagnosis of diseases of the heart and great vessels. 9th ed. Boston, MA: Little, Brown; 1994.

17. Sanders MH, Black J, Costantino JP, Kern N, Studnicki K, Coates J. Diagnosis of sleep-disordered breathing by half-night polysomnography. Am Rev Respir Dis. 1991;144(6):1256-61.

18. Khawaja IS, Olson EJ, van der Walt C, Bukartyk J, Somers V, Dierkhising R, et al. Diagnostic accuracy of split-night polysomnograms. J Clin Sleep Med. 2010;6(4):357-62.

19. Lohman TG, Roche AF, Martorell R. Anthropometric standardization reference manual. Champaign: Human Kinetics; 1988.

20. Johns MW. A new method for measuring daytime sleepiness: the Epworth sleepiness scale. Sleep. 1991;14(6):540-5.

21. Carter R, 3rd, Watenpaugh DE. Obesity and obstructive sleep apnea: Or is it OSA and obesity? Pathophysiology. 2008;15(2):71-7.

22. Resta O, Foschino-Barbaro MP, Legari G, Talamo S, Bonfitto P, Palumbo A, et al. Sleep-related breathing disorders, loud snoring and excessive daytime sleepiness in obese subjects. Int $\mathrm{J}$ Obes Relat Metab Disord. 2001;25(5):669-75.

23. Rabec C, de Lucas Ramos P, Veale D. Respiratory complications of obesity. Arch Bronconeumol. 2011;47(5):252-61.

24. Crummy F, Piper AJ, Naughton MT. Obesity and the lung: 2. Obesity and sleep-disordered breathing. Thorax. 2008;63(8):738-46.

25. Guilleminault C, Stoohs R, Kim YD, Chervin R, Black J, Clerk A. Upper airway sleep-disordered breathing in women. Ann Intern Med. 1995;122(7):493-501.

26. Redline S, Kump K, Tishler PV, Browner I, Ferrette V. Gender differences in sleep disordered breathing in a 
community-based sample. Am J Respir Crit Care Med. 1994;149(3 Pt 1):722-6.

27. Sakakibara H, Tong M, Matsushita K, Hirata M, Konishi Y, Suetsugu S. Cephalometric abnormalities in non-obese and obese patients with obstructive sleep apnoea. Eur Respir J. 1999;13(2):403-10.

28. Li KK, Kushida C, Powell NB, Riley RW, Guilleminault C. Obstructive sleep apnea syndrome: a comparison between Far-East Asian and white men. Laryngoscope. 2000;110(10 Pt 1):1689-93.

29. Chierakul N, Chaipattarapol C, Ruttanaumpawan P, Nana A, Naruman C, Tangchityongsiva S. Comparison of clinical and polysomnographic characteristics of non-obese and obese patients with obstructive sleep apnea. J Med Assoc Thai. 2007;90 (Suppl 2):48-53.
30. Davies RJ, Stradling JR. The relationship between neck circumference, radiographic pharyngeal anatomy, and the obstructive sleep apnoea syndrome. Eur Respir J. 1990;3(5):509-14.
Correspondencia: Jorge Rey de Castro.

Dirección: Av. Alfredo Salazar 314, San Isidro, Lima 27, Perú. Telefax (511) 2219013

Correo electrónico: jorgerey@rcp.net.pe.

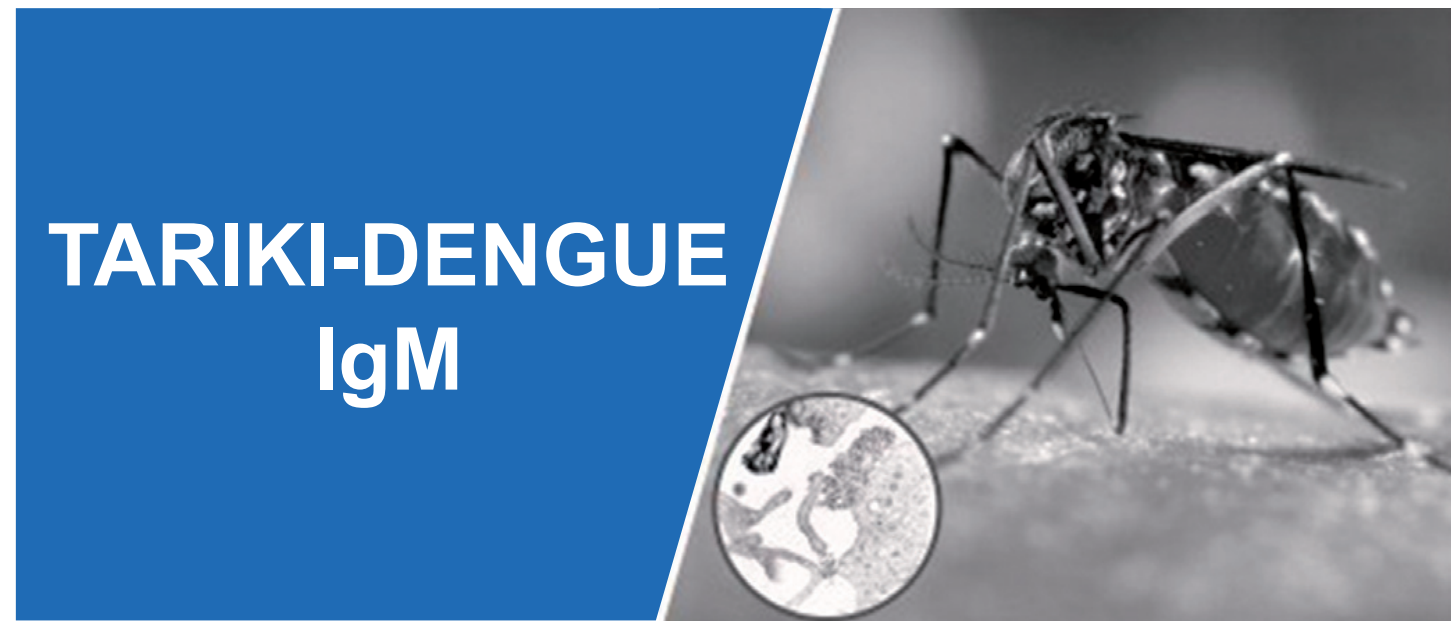

\section{ELISA de CAPTURA IgM DENGUE}

INSTITUTO NACIONAL DE SALUD

LIMA - PERÚ

\section{Pronto disponible en el Perú....}

\section{Kit para la determinación de anticuerpos IgM anti Dengue, desarrollado y producido por el Instituto Nacional de Salud. INS/MINSA}

